\title{
The climatic, constructional, and cultural primacy of the envelope: UR22, a case study
}

\author{
V. Snyder \\ University of Texas at Austin, USA
}

\begin{abstract}
More than any other singular aspect, a building's envelope has the capacity to integrate many of the primary concerns of any new building design into a unified, harmonious artifact. UR22 (a residence built in 2008 in suburban Dallas) is presented as a case study that demonstrates how direct and synthetic responses registered by a building's envelope can successfully operate within multiple contexts - climatic, constructional, and cultural. Regionally, UR22 represents a shift from the excessive land and energy consumption and social alienation of typical contemporary American residential developments. UR22 is demonstrative of the client's intention for a greater sense of community and respect for the environment and energy at the scale of the development, site, and the home itself. Quantitatively, UR22 has achieved LEED-H GOLD and Energy Star HERS 50 (50\% energy reduction) ratings. Qualitatively, UR22 remediates and exploits a potential conflict between comfort and some very direct expressions of natural forces/energies both resisted and gathered within the harsh climate of the North Texas. This paper presents how UR22 is consistently organized and designed to produce an integrated form with respect to site orientation, protective enclosure, control of the elements and structure.
\end{abstract}

Keywords: climate, construction envelope, culture, integrated form, solar orientation, enclosure, collected elements, structure.

\section{Introduction}

All architectural projects must address multiple concerns and most often a singular factor becomes the dominant "driver" from which other decisions must attempt to locate their relevancy. Certainly, within the context of a volatile climate such as in North Texas, the potential solar impact for any project could 
indeed be the most influential concern, as is the case presented here as an expansion of an earlier paper by this author [1]. However, there are many other factors which must be integrated. Moreover, the term integration has many meanings even if the definition is constrained within a more narrowed scope located within the field of building technologies. Integration here simply refers to what many consider to be the primary task of an architect. Architects have been educated and trained to assimilate vast amounts of largely unrelated information into a building artifact that fuses both quantitative data and qualitative characteristics. UR22 is presented here as a case study which: 1seeks to extend a recurring notion that architecture is a medium whereby the complexities inherent in any contemporary construction project can be synthesized into a unified, coherent, and harmonious artifact recounting such observations similar to Wittkower's [2] and, 2- from Evans [3] as an art form, architecture is capable through resemblance to be in a special position to resolve disparate and often contradictory concepts attached to those complexities.

In addition to the general design of the building which also includes the selection and placement of numerous products and components, architects would also prefer the opportunity to be part of a project team involved in the actual design and development of much of the products and material configurations. This is not a desire out of conceit or a merely visual issue but a concern that as many parts as possible should have a resonating dialog with each other. To that, historical regional precedents - that demonstrate the intersections between these various demands particular to a given project - were closely examined. A search to unearth shared architectural expressions between the climatic, constructional, and cultural contexts is the real impetus towards positing an innovative architectural design solution within this region. The design of the residence UR22 was undertaken to explore such possibilities.

\subsection{General site}

UR22 is a residence located in the Urban Reserve; an ecologically sensitive, zero-lot line single-family housing development in northeast suburban Dallas (50 lots in 13 acres) 15 minutes from the city's center. Commissioned by the developer, this project is one of the first homes built within the new community. Most importantly, it is demonstrative of the client's intention for a greater suburban density, sense of community and respect for the environment at the scale of the development, building site, and the home itself. Storm water recycling and filtration with rain gardens \& retention ponds along with native, non-invasive, low-water use plantings were used throughout the community.

\subsection{Program}

Briefly, the program for UR22 is targeted for those whose children are grown but return to visit periodically. The program also reflects a high degree of spatial connectivity, internally and externally. Contrary to this, family or overnight guests are provided with a strong sense of privacy, despite the overall open spatial quality. Another priority is a variety of entertainment spaces that can 
accommodate both small gatherings and large groups. Lastly, given the intended identity of the development and following the qualities of the master-plan, low maintenance materials and energy conservation are essential mandates.

\subsection{Climate}

Climate in the Dallas area is characterized as a humid-temperate region but repeatedly winds with cooler, drier air come from the north and west to reduce or remove humidity. The typical temperature extremes are from about $105^{\circ} \mathrm{F}$ $\left(41^{\circ} \mathrm{C}\right)$ to $20^{\circ} \mathrm{F}\left(-7^{\circ} \mathrm{C}\right)$ with average temperature highs ranging from mid-to-upper $90 \mathrm{~s}$ in July to the mid-50s in January. A $20^{\circ} \mathrm{F}\left(11^{\circ} \mathrm{C}\right)$ diurnal drop at night is common throughout the year. Annual precipitation is in the mid-to-upper $30 \mathrm{~s}$ (inches) with usually at least some snow fall, although it may only amount to a few inches. Generally, the potential for cooling breezes in the summer may come either from the Gulf of Mexico in the south or from the plains to the north. Often during the fall and winter, strong cold fronts known as "Blue Northers" that originate from Canada, roll down the Great Plains region of the central US reaching Dallas and drastically reduce the temperature within a couple hours $-\mathrm{a}$ $30^{\circ} \mathrm{F}\left(17^{\circ} \mathrm{C}\right)$ shift is not unusual. Frequently, warm Gulf and cold Canadian fronts collide in a larger region referred to as "Tornado Alley" within which Dallas is also located. In other words, North Texas is a region with great temperature swings and weather unpredictability. Obviously, the building must be agile to adapt to these extremes.

\subsection{Construction}

Construction methods and materials within the development mirror typical American residential assemblies which generally prioritize speed and economy over durability and craft. Rapid wood frame construction with gypsum board interiors and exposed concrete floors are common to the majority of projects within this new community. To address and potentially exploit this reality, the project naturally aims to conjoin the design logic with the constructional techniques regularly used in the area.

\subsection{Culture}

Culturally, the region is predictably complex despite the presuppositions about Texan culture in general and Dallas specifically. Rather, the cultural aspects concerned here are those embedded within the historical architectural contexts of climate response cited in this paper as well as the contemporary expectations of the client and prospective buyer with regard to typical home construction in the area.

\section{Precedents}

The awareness of the significance of site orientation by those constructing buildings is clearly visible in many of the $19^{\text {th }}$ century and early $20^{\text {th }}$ century historical precedents taken from several areas of Texas and the other regions of 


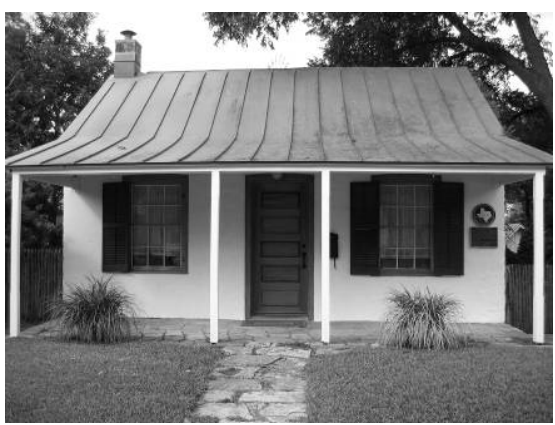

Figure 1: 19c, Sunday house, Figure 2: Fredericksburg, TX.

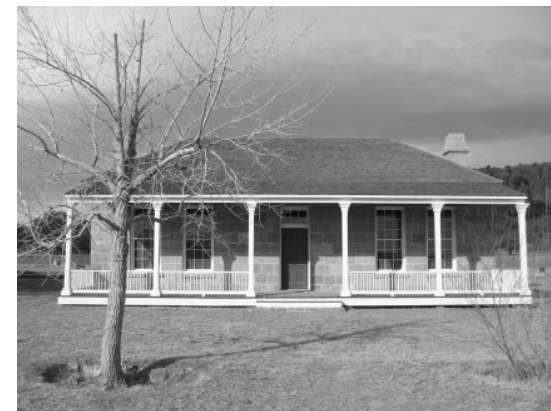

$19 \mathrm{c}$, Officers' quarters, Fort Davis, TX.

the United States (Figs. 1,2). These examples demonstrate the empirical understanding of earlier immigrants and inhabitants as to the efficacy of buildings elongated along the east-west axis to optimize solar behavior. This fact has also long since has been proven by quantitative models starting with Olygay [4], but unfortunately has been largely ignored by post-WWII housing developments in the US where typically replicated designs are produced without regard to site orientation.

Returning to the precedents, we can compare the shapes of the building envelopes. While elongation of simple volumes is consistent, we also notice that the major elements of entry, fenestration, heating element, roof and wall harmonize into powerful, candid overall forms. While much of the configurations and materiality are program and climate dependent, all of the examples provide tangible existential and emotional protection as well. For example, the porches continue the pre-air conditioning tradition of a gathering space for social interaction due to their protection from the elements of weather. They also meditate between the public realm and the private realm of one's own home. The building's materiality, mostly related to issues of durability, also contribute to the concept of protection.

\subsection{Materials}

Readily abundant limestone was found by early settlers in the Texas Hill Country located in the central part of the state. Due to its soft nature, new immigrants fashioned thick walls for stability and durability. Often this material choice is advantageous because of its mass to regulate diurnal swings (Figs. 1, 2). However, Central Texas (as with North Texas) also experiences periods of high humidity where a lighter construction assembly is also acceptable and at times preferable to a heavier construction. Stamped metal cladding also provided a lightweight, stable surface in early construction and other thin gauge metal applications were used for roofing. In fact, because of custom and resilience, standing seam metal roofs are still the preferred choice today in much of Texas. Adobe brick and sandstone are also used traditionally in West Texas. 
In this arid climate, a more massive material choice can take full advantage of the diurnal swing.

\subsection{Protective enclosure}

The protective aspect of a building would seem to be quite obvious but its fundamental ability to provide a sense of well being is frequently undermined in contemporary buildings, particularly those that privilege technology as an aesthetic for progressive design. In contrast, Picasso's painting, Françoise, Claude and Paloma, 1954 (Fig. 3) illustrates the emotional and existential simplicity of a larger protective form as represented by the over-arching mother who provides the watchful space for the play and embrace of her children. All of the figures are in concord.

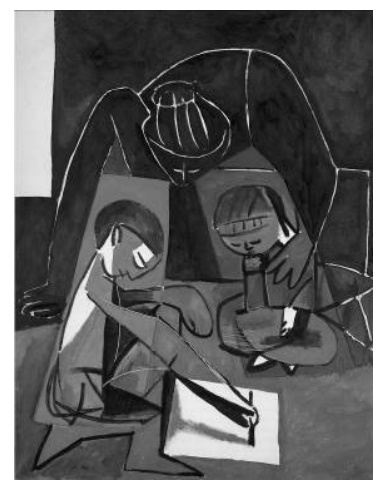

Figure 3: $\quad$ Picasso, Françoise, Claude and Paloma, 1954.

\subsection{Controlling elements}

Despite their regional differences, the $19^{\text {th }}$ century buildings shown also exhibit the potential for a directness and synthesis in building by which sun, wind, and precipitation are controlled, to be either gathered or repelled depending upon seasonal requirements. Here the importance of the roof configuration is quite apparent. The materials used on a roof or wall usually vary and so one may see them as quite separate entities. The choice of different materials was usually done for reasons of cost and material availability but a change in materials also acknowledges the variation of material and assembly effectiveness due to a horizontal versus a vertical application. Technical and detailing developments have changed this whereby many contemporary buildings exhibit the use of similar materials for both roof and wall applications that can be achieved without monetary penalty. Although this practice of using the same material for the wall and roof began its use in more contemporary times as an aesthetic rather than strictly functional practice, this approach often reduces costs related detailing and trade coordination. 
Regardless of material continuity, the roof configurations shown in the precedents result in multi-functional elements such as the porch which demonstrates a simultaneous protection from sun and rain combined with a purposeful collection of water and breezes. Again, we are attracted to the honesty and economy of effort in these examples which operate successfully on multiple levels concurrently.

\subsection{Structure}

Besides the briefly aforementioned topics of solar orientation, protective enclosure, and the collection of weather elements, the constructional assemblies of these early structures are also noteworthy. Organizationally, repetition of shape and detail was necessitated by limited supplies of materials, gaps in the knowledge of construction, and an immediate desire for safety and protection which therefore required that the newly arrived settlers expedite construction assembly and reconsider expectations of quality. This produced a condition that closely resembles the state of construction today with regard to the issue of speed. Intellectually, a repetition of structure left exposed provides a conceptual and physical datum that registers the human body within the building. Emotionally, the dynamic forces of gravity and wind resisted are physically articulated and therefore lend themselves to a kind of natural or unintended decoration through fascination and replication (Fig. 4).

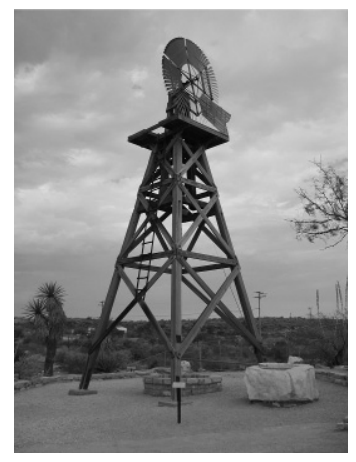

Figure 4: $\quad$ Eclipse windmill 1900, gantry, TX.

\section{UR22, a case study}

After searching for an appropriate response to the many circumstances required for a design of a residence in Dallas that is reflective of its time, a methodology emerges from the recurring categories of solar orientation, protective enclosure, collected weather elements, and structure as found latent in the $19^{\text {th }}$ and early $20^{\text {th }}$ century historical structures alluded to here. Through resemblance, formal and systemic strategies are deployed to produce an interconnected construction that ties the past to the present beyond simple duplication. 


\subsection{Site orientation of UR22}

As an intentional refutation of the indifference to site orientation common in most American developments, the master-plan of the Urban Reserve development situates most of the property lots along the east-west axis with an approximate elongation ratio of about 3:1 - a length of about 50' $(15 \mathrm{~m})$ and a width of $150^{\prime}(45 \mathrm{~m})$. In an effort to increase density, tract widths are narrowed relative to typical tracts found in suburban Dallas. Building-to-lot relationships are controlled by a zero-lot line policy where homes must be located on the northern property lines. This placement, combined with the solar orientation of the site, results in restrictions that impact the design in several ways: 1) Direct views into the adjacent property to the north are forbidden for matters of privacy, 2) Overhangs along the north property line are not possible due to their location, 3) A 20 ' $(6 \mathrm{~m})$ wide minimum south yard is required, 4) The building's west edge must directly adjoin the community retention pond, and 5) Building heights are limited by code to $28^{\prime}(9 \mathrm{~m})$ above grade. These last two create the potential problem where solar access to a building's interior in the winter months, when most desired, may be blocked by the neighboring home.

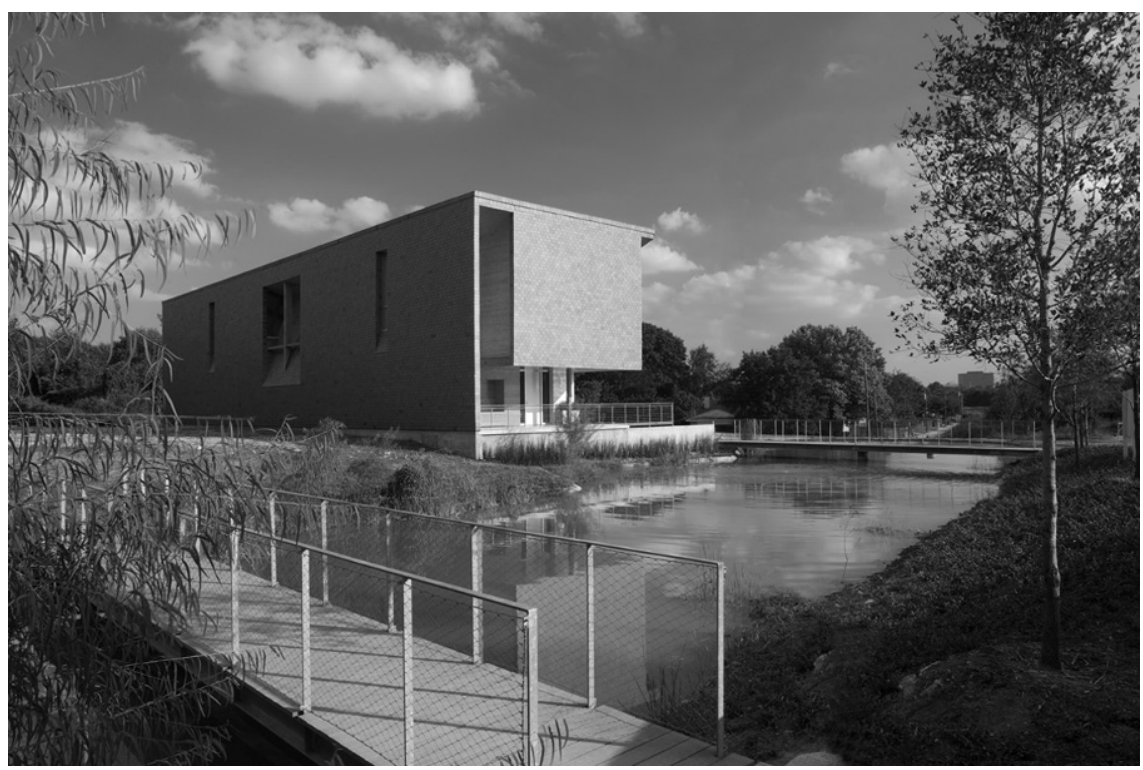

Figure 5: View from NW, UR22, Dallas, TX.

Due to the restricted site conditions cited, UR22 (Fig. 5) results in an overall building volume that is narrow and elongated along the east-west axis, a north wall with limited fenestrations which are inset and placed high, substantial overhangs on the east-south-west sides and a predominant roof slope that is inclined to the south. The much narrower east and west facades are also generally opaque and when coupled with the programmatic solid volumes (such 
as bathrooms and garages) they provide additional solar blockage to prevent unwanted heat gain on those typically problematic sides. These protective elements allow for a large expanse of south facing glass. This enhances the interior/exterior connection to the narrow south yard while supplying the interior with generous amounts of indirect lighting throughout the day (Fig. 6).

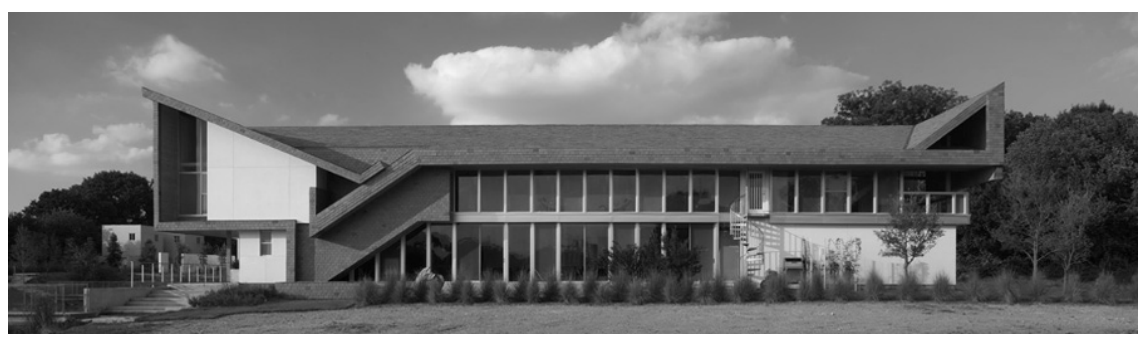

Figure 6: South elevation, UR22, Dallas, TX.

\subsection{Protective enclosure of UR22}

As recognized from the precedents, the envelope may possess both volumetric and surface protective qualities. UR22's durable slate envelope does exactly this. The cladding assembly simultaneously creates a temperature differential for enhanced ventilation and the overall building envelope is precisely configured (with deep overhangs, proper orientation between the vertical and horizontal planes) to control the environmental elements and thereby provide an embracing sense of enclosure that mediates between the inside and outside. The solid volumes and stair elements screen the interior from the public entry and alley approaches while reinforcing the notion of these objects as protected entities that are in a harmonious dialog.

While cladding an entire building in slate has been a common practice in Europe for centuries, the application of slate on both the roof and walls of a single project it is largely unused in the United States. The primary envelope of UR22 is clad with a light-colored slate for several environmental reasons (although slate attributes have yet to be recognized by LEED): 1- Durability; when properly installed Grade S1 slate will last well over 100 years. 2- No toxic waste; with no chemicals released into the environment and no landfill waste with regard to life cycle issues (since it is natural rock), slate can be reused for both building and landscape applications. 3- Breathable wall and roof surfaces; temperature differential created by the solar gain of the slate induces vertical air movement when an air space is provided directly behind the slate. This results in cooler interior surfaces and will also evaporate any moisture that may inadvertently penetrate the surface of the assembly. Double lapping (head-lap) results in good water resistance as well as produces a very breathable surface in the slate assembly when mounted on vertical furring; a necessity in the hot North Texas climate. 4- Elimination of all roof penetrations; aside from being visually clean, this provides for an obstacle-free roof surface for future easy installation of photovoltaic or solar panels. This area for future placement 
resulted from a desire to provide a specific location that is screened from the primary entry directions of either the front visitor's entry from the main street or from the alley access. The east and west triangular prismatic volumes and the upturned "gutter" delineate the placement area by serving as a frame for the panels (Fig. 7).
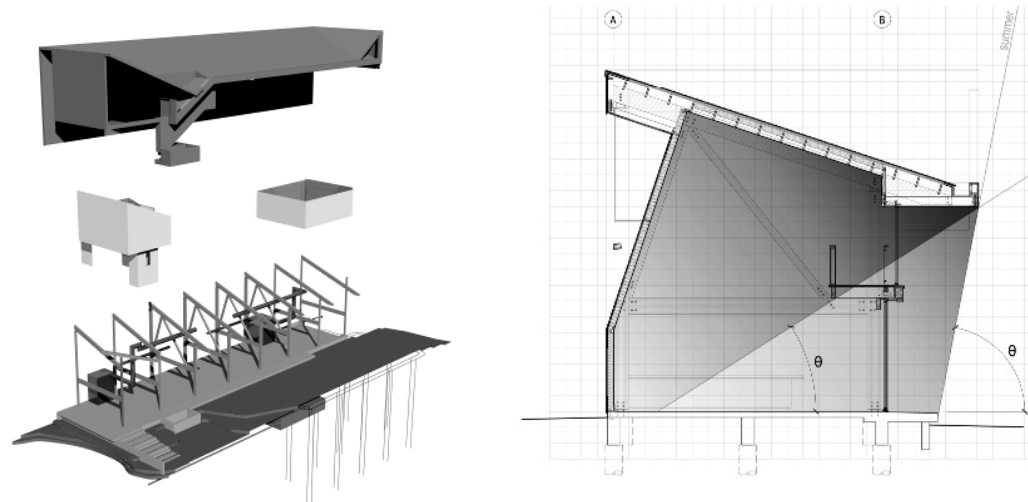

Figure 7: Organization,

UR22, Figure 8:

Solar angles, UR22, Dallas, TX.

Dallas, TX.

\subsection{Collected weather elements of UR22}

The elements of rain, sun, and wind are controlled primarily through the envelope configuration. Formal overlaps resulting from the exigencies of collection or diversion needed to control the weather elements were aggressively pursued and determined through both graphic geometrical and digital means. Through envelope shaping and volume placement, sunlight is either blocked or enters the building dependent upon seasonal requirements. The independent, extended nature of the slated envelope not only creates a range of shaded spatial enclosures but also draws together breezes for enhanced natural ventilation. Door and window placement is crucial. Primary natural ventilation is achieved though multiple openings in the expansive glazed facade to capture prominent breezes from the south. Exit openings are located in the "slots" of the slate clad envelope on west and east facades to produce cross-ventilation instead of direct passage which would result in many areas with little or no air flow as illustrated from Boutet [5].

Digital solar studies were used to determine sizing, location, and effectiveness of specific variations in the envelope (e.g. the design of the "flaps" and "boxes" on the SE and SW corners) to mitigate heat gain in the long summers of North Texas while permitting solar gain in the relatively shorter winter months. In fact, the sun remains largely outside of the building interior between April and September (Fig. 8). In the winter, thermal lag from sunlight directed onto the exposed concrete floor on the ground level is used advantageously. Additionally, the extensive, reflected daylight that enters the building through 
the expanse of the window wall on the south creates bright, lofty interior spaces thereby reducing artificial lighting loads. Although the digital modeling had a direct effect on the formal subtleties of the envelope shaping on the east-west ends, most heat gain is controlled by the design of the south overhang since the transverse section represents a large portion of the house. As cited earlier, the proximity of the homes to one another may greatly affect their ability to acquire sunlight into the home during the winter months when the additional heat is welcome. UR22 attempts to reduce this impact by using the window wall and placing the glazing as high as possible. Therefore, this also results in a substantial overhang to combat the summer sun. Not only is this condition expressed honestly but it is used in conjunction with the rainwater collection system.

As with many current sustainable activities, rainwater collection is a return to previous practices that reflect a simplicity and straightforwardness. Rainwater is collected from the entire roof area and this dynamic is expressed as a "moment of collection" to a single fountain at the base of the enclosed stair with any overflow stored in a cistern. The shape of the envelope expresses this collection in conjunction with the slope of the stair and represents a visual stability of the dominant envelope, thereby representing one example of the notion of integrated systems that go beyond the functional.

\subsection{Structure of UR22}

As with the other categories, the deployment and configuration of UR22's structure is consistent with the historical precedents. The organization and modulation of the house is clearly visible as articulated by the exposed glu-lam frame structure. An all-wood, fast growth, southern pine structure (exc. foundation and connectors) is the primary and secondary construction material selected by both the architect and the client for its workability, availability, and speed of assembly (Fig. 9). Southern pine was also used for all exterior soffit and interior upper level flooring to strengthen the notion of using a limited palette of materials as shown in the precedents.

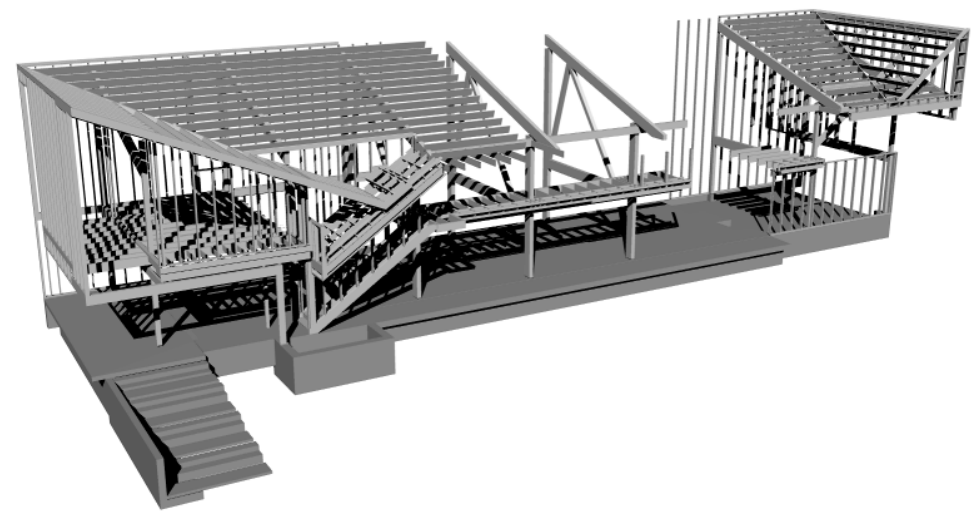

Figure 9: Architect's digital structural model, UR22, Dallas, TX. 
For conceptual clarity and organization, the entire glu-lam and framing layout was also digitally modeled and initially configured by the architect (Fig. 9) to communicate more effectively with the contractor and structural engineer (the party responsible for verifying each component and systemic correctness). This 3-dimensional modeling allows for greater manageability of the structural logicto-architectural design relationship and for enhanced quality control given the modestly priced framing and construction practices of the Dallas market. Furthermore, the repetitive spacing of the major frames at 16' on center aids in quality control by assuring that reference lines for construction are never more than 8 ' away.

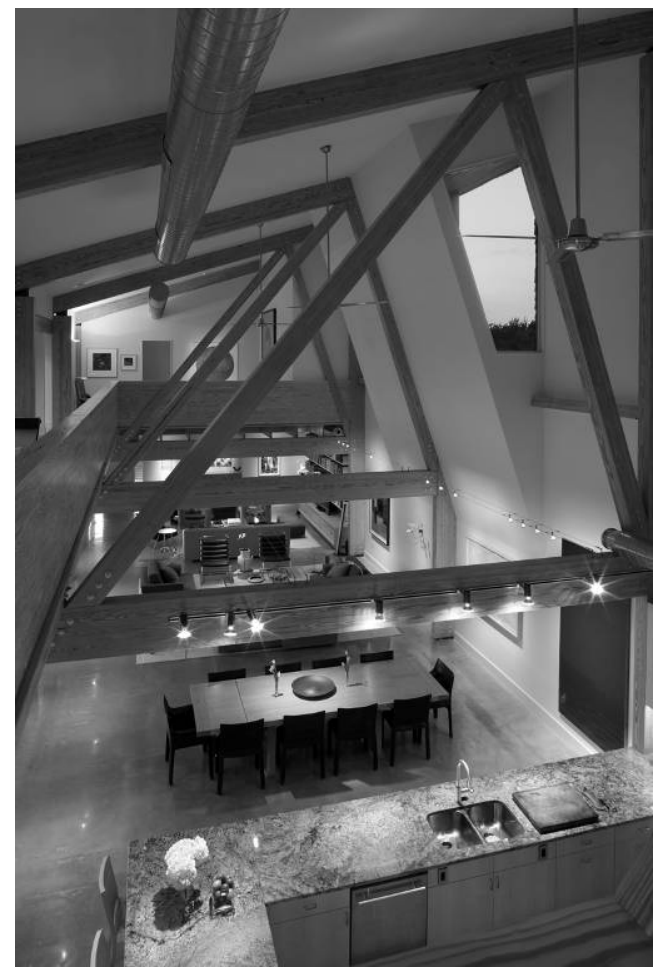

Figure 10: Glu-lam Frames, UR22, Dallas, TX.

Additionally, OVE (optimum value engineering) framing techniques were used to reduce wood consumption. The digital modeling also assists in visualizing this cost and material savings approach since OVE largely relies on a more precise alignment of the vertical to horizontal components than used in traditional light framing.

The heavier, cadenced glu-lam frame construction also employs recurring diagonal bracing to provide exceptional lateral strength and directly express the powerful forces of wind and gravity resisted (Fig. 10). As noted earlier, exposed 
wood structures tend to impart a natural ornamentation through their repetition and material warmth.

\section{Conclusions}

Conceptually a building's envelope has the capacity to integrate many of the primary concerns of any new building design into a unified, harmonious artifact. Quantitatively, UR22 has achieved LEED-H Gold and Energy Star HERS 50 (50\% energy reduction) ratings - independently verified. Qualitatively, UR22 represents a planar roof/wall system, made of a durable, ventilation-inducing envelope that is manipulated to remediate and exploit a potential conflict between comfort and the harsh climate, constructional challenges, and complex cultural contexts in North Texas (Fig. 11).

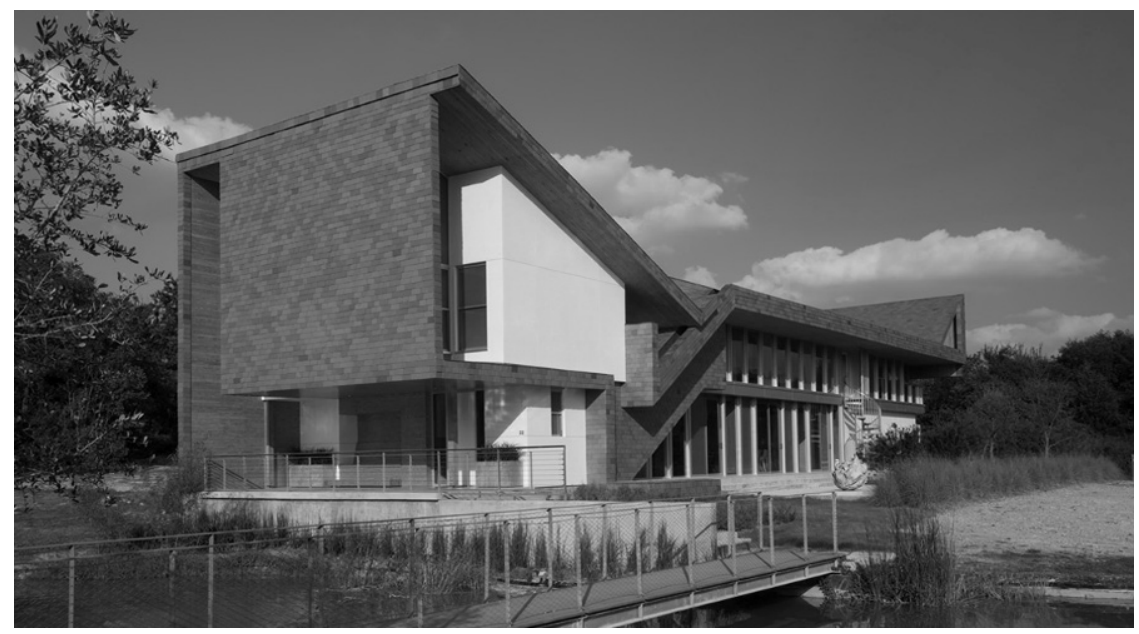

Figure 11: View from SW at entry, UR22, Dallas, TX.

\section{References}

[1] Snyder, V., Integrated Form in North Texas, Solar Architecture \& Urban Planning Conference Proceedings, Munich, pp.115-119, 2009.

[2] Wittkower, R., Architectural Principles in the Age of Humanism, W. W. Norton \& Company, New York and London, 1971, 1962.

[3] Evans, R., The Projective Cast, MIT Press, Cambridge, MA, pp. 25-27, 1995.

[4] Olygay, V., Design With Climate, Princeton University Press, Princeton, pp. 87-90, 1967

[5] Boutet, T., Controlling Air Movement: A Manual for Architects and Builders, McGraw-Hill, New York, p. 87, 154, 1987 